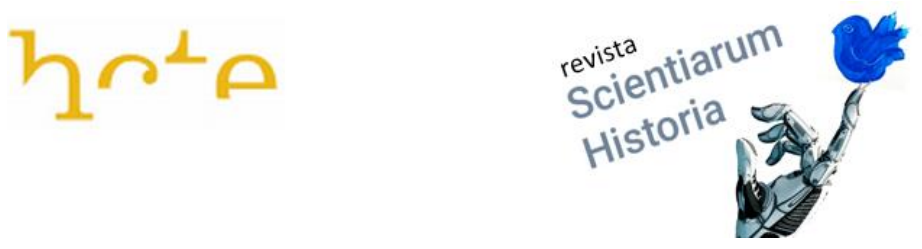 \\ Rede psi do bem: a collaborative network supported by the use of technologies during the pandemic (Covid-19)
}

\section{Rede psi do bem: uma rede colaborativa apoiada pelo uso de tecnologias durante a pandemia (Covid-19)}

\author{
Maria de Fátima Rezende Francisco ${ }^{1}$, Marcela Oliveira França ${ }^{4}$, Angélica Fonseca \\ da Silva Dias ${ }^{2,3}$, Juliana Baptista dos Santos França ${ }^{5}$,Mary Uchiyama Nakamura ${ }^{1}$ \\ ${ }^{1}$ Universidade Federal de São Paulo/Escola Paulista de Medicina-UNIFESP/EPM \\ ${ }^{2}$ Programa de Pós-graduação em História das Ciências e das Técnicas e Epistemologia, \\ Universidade Federal do Rio de Janeiro \\ ${ }^{3}$ Instituto de Aplicações e Pesquisas Computacionais-NCE, da Universidade Federal do \\ Rio de Janeiro \\ ${ }^{4}$ Bacharelado das Ciências Matemáticas e da Terra-BCMT/UFRJ \\ ${ }^{5}$ Universidade Federal Rural do Rio de Janeiro-UFRRJ \\ mfatimafrancis@globo.com,mar-olifranca@hotmail.com, angelica@nce.ufrj.br, \\ julibsf@gmail.com,maryuchiyama0@gmail.com
}

Recebido: 27/04/2021 Aceito: 29/04/2021 Publicado: 04/05/2021

DOI: $10.51919 /$ revista_sh.v1i0.307

\begin{abstract}
The Covid-19 pandemic and social isolation generated multiple effects on the environment, public health, socioeconomic indicators and, mainly, on humans. The decrease in the socio-affective relationship and the distance brought individuals a great difficulty in interacting, generating devastating effects. This investigation was intended to promote transformation in the digital environment. To this end, daily videos were created creatively during the current pandemic. The videos were composed of the participation and testimonies of specialists, children and professionals from different areas. The study aims to observe the reactions and exchanges of affection on social networks from the perspective of specialists, as well as to identify how the use of technological tools is perceived by individuals. We also seek, through this project, to understand the relations of the integration of the inter / transdisciplinary knowledge of Psychodynamics with Human Aspects in Computer Science. The research acted in bringing individuals together and interacting to build a network of affection, expanding the capacity to feel the world and the integration of human beings with technology.
\end{abstract}

Keywords. Mental Health and Covid-19. Perception in collaboration. Psychology of emoji.

Resumo. A pandemia do Covid-19 e o isolamento social gerou múltiplos efeitos no meio ambiente, na saúde pública, nos indicadores socioeconômicos e, principalmente, no ser humano. A diminuição da relação socioafetiva e o distanciamento trouxeram para os 
indivíduos uma grande dificuldade de interação provocando efeitos devastadores. Esta investigação teve como intenção promover a transformação diante do meio digital. Para isso, foram construídos, de forma criativa, vídeos diários durante a atual pandemia. Os vídeos foram compostos por participações e depoimentos de especialistas, crianças e profissionais de diversas áreas. Este estudo visa a observação das reações e trocas de afeto nas redes sociais sob a ótica dos especialistas, bem como identificar de que forma o uso das ferramentas tecnológicas são percebidos pelos indivíduos. Também buscamos, através desse projeto, entender as relações da integração do conhecimento inter/transdisciplinar da Psicodinâmica com os aspectos humanos na Computação. A pesquisa atuou na aproximação e na interação dos indivíduos para a construção de uma rede de afeto ampliando a capacidade de sentir o mundo e a integração do ser humano com a tecnologia.

Palavras-chave: Saúde Mental e Covid-19. Percepção na colaboração. Psicologia dos emojis.

\section{Introdução}

A Organização Mundial de Saúde (OMS, 2020), dia 11 de março de 2020 , anunciou que a Covid-19 é uma PANDEMIA e passa a constituir uma Emergência Pública de Importância Internacional. E a quarentena foi a forma encontrada para enfrentar a contaminação em massa pelo Sars-COV-2 ( Covid19). O vírus teve seu início na China e se mostrou veloz na contaminação e fortemente letal , chegando rapidamente a várias regiões do mundo.

No mundo todo a restrição da movimentação das pessoas no combate a disseminação do vírus trouxe muito sofrimento psíquico para aqueles que moram sozinhos ou não, tédio, medo da morte, rituais de luto suspensos, exaustão profissional, Burnout, estresse pós traumático, insônia, frustração e culpa pela morte de tantos pacientes e de amigos (Xiang et al, 2019). Neste contexto, sintomas psicológicos começam aparecer nos pacientes e seus familiares no momento do diagnóstico de Covid-19, fazendo tanto o doente como seus familiares e a equipe de saúde sofram com desordens psíquicas graves.

A partir destes cenários e com a necessidade de atender e apoiar pacientes e amigos foi construído o projeto de pesquisa Rede Psi do Bem. Esta rede colaborativa foi embasada na teoria da psicologia de Winnicott (1971), que afirma que viver a criatividade constitui um estado saudável e necessário. Baseados nesse conhecimento, utilizamos o veículo de vídeos informativos composto pelas participações e depoimentos de especialistas, crianças e profissionais de diversas áreas, disparados diariamente, pelas redes sociais Whatsapp, Facebook e Instagram.

Este trabalho propõe uma análise por observação das reações dos indivíduos a partir dos vídeos, a fim de identificar as trocas de afeto realizadas e o estado emocional provocado. Para isso, foi conduzido um estudo exploratório das redes sociais para entender "Como o uso de mídias sociais, em uma rede colaborativa, podem apoiar a saúde mental dos individuos em situações de isolamento?". Foram coletadas reações através de mensagens e emojis postadas nas redes sociais (Sternbergh, 2014). A pesquisa é relevante pelo contexto desafiador trazendo contribuições na saúde física e mental dos indivíduos. 
Este artigo está organizado em cinco seções. A seção 2 apresenta trabalhos relacionados ao isolamento social, conceitos da psicologia e as redes colaborativas. A seção 3 apresenta a abordagem sobre o projeto Rede Psi do Bem e suas características. Seguindo isso, Seção 4 apresenta a discussão e avaliação da pesquisa. Para terminar este artigo, a Seção 5 apresenta nossas conclusões que evidenciam os objetivos alcançados e as limitações desta pesquisa.

\section{A Psicologia e as Redes Colaborativas}

A contribuição da psicologia no contexto de pandemia é enorme, pois se aplica em hospitais no atendimento a pacientes, suas famílias e a equipe de profissionais que estão exaustos com a demanda de trabalho vivendo momentos de pânico, medo, estresse, depressão e ansiedade (Sá, 2018). O atendimento individual foi suspenso, tendo os profissionais terem que atender de forma remota para evitar a contaminação pelo Covid19 (Conselho Federal de Psicologia, 2018).

Em virtude do isolamento os indivíduos foram obrigados a ficar em suas casas. Este novo modelo não permitiu uma preparação prévia para este novo estado. Kossek (2016) destaca em seus estudos a necessidade de limites para separar a rotina de trabalho e de casa. Estes limites, ocasionados pelo isolamento, podem ser: (i) De espaço - definir locais físicos na casa para trabalhar; (ii) De tempo - estabelecer uma rotina horária que faça melhor uso do seu nível de energia, de produtividade, e com tempo para viver sem estar trabalhando; (iii) De tecnologia - usando certos dispositivos (laptops ou celulares, por exemplo) só para trabalhar, e outros para relaxar; (iv) Psicológico - Roupas, acessórios e hábitos que te ajudam a diferenciar se você está em modo de trabalho ou não.

Duan e Zhu (2020) apresentam resultados sobre os efeitos do coronavírus na saúde mental, indicando um grande aumento do estado de depressão, ansiedade, estresse, transtorno de pânico, insônia, medo e raiva. Os autores também relatam que os problemas psicológicos mudam e as intervenções devem ser direcionadas e adaptadas conforme as demandas que forem sendo apresentadas. Lima et al (2020) afirmam que a maioria dos profissionais de saúde que trabalham nas emergências não recebem treinamento para os cuidados com a saúde mental. Este fato pode agravar ainda mais o estado físico e mental dessas pessoas (Schmidt, 2020).

Para Winnicott (1975), viver a criatividade constitui um estado saudável e a submissão é a base doentia da vida. $\mathrm{O}$ autor ressalta que o espaço só acontece em relação a um sentimento de confiança. Para isso é necessário que exista um canal, ou seja, "o lugar em que está localizada a experiência cultural é o espaço potencial entre o indivíduo e o ambiente". Este espaço precisa ser construído para gerar confiança e resgatar experiências vividas.

A Teoria de Malan (1983) apresenta os triângulos de sentimentos. O primeiro triângulo consiste na explicação de como estávamos usando as nossas defesas, ansiedade e qual o sentimento encoberto frente ao fenômeno. Segundo o autor, a semelhança entre todas as ciências é efetuar o trabalho a partir da observação, para que se tornem inquestionáveis quando comprovadas. Já o segundo triângulo investiga sentimentos do passado, geralmente com relação aos pais, trazendo-os para o aqui e agora, chamado de triângulo do tempo. Esta análise pode ser trazida para situações presentes e resgatadas a partir da observação do fenômeno. 
O Covid-19 trouxe um cenário desconhecido, além de novos fenômenos dentro dos grupos que merecem ser observados. Para Winnicott (1978) entender os fenômenos e construir conexões criativas pode apoiar os indivíduos. Será que os vídeos através de redes colaborativas podem trazer o apoio e entendimento dos impactos do Covid-19 encontrados nos indivíduos que estão em diferentes lugares, como: hospitais, casas, escolas ou mesmo trabalho? Será que as respostas vindas através dos emojis não estão nesse espaço potencial e de confiança que cada indivíduo tem do uso do objeto percebido na mensagem? Os emojis são símbolos usados para transmitir sentido em determinados contextos de interação, mas, ao mesmo tempo, fazendo emergir sentidos acrescidos de muitos outros significados, especialmente de emoções.

\section{Projeto Rede Psi do Bem}

A motivação para a criação da Rede Psi do Bem tem origem na experiência de um dos autores, que nos últimos 38 anos vem atuando no atendimento psicológico à saúde mental. Com o Covid-19 os atendimentos e outras demandas foram colocadas em situação de emergência e crise em função do isolamento social. A partir do confinamento prolongado pela quarentena e, na tentativa de acalmar a ansiedade evidenciada, foi necessário o distanciamento respaldado por estudos científicos no combate ao Covid-19. Sem apoio jurídico e científico e com uma necessidade de entender e informar o que estava acontecendo no mundo, foram utilizadas mídias sociais para o envio de vídeos informativos. Estes artefatos tratavam sobre os cuidados com a saúde física e mental e reflexões sobre os impactos do COVID-19 no Brasil e no Mundo.

Os vídeos foram produzidos de forma criativa possibilitando apoiar os indivíduos, possibilitando ao observador um estado para apoiar os participantes da Rede a sair do seu padrão de reação ao imprevisível. A ideia principal foi usar recursos internos e outras formas de pensar, na tentativa de amenizar a ansiedade dos participantes da Rede Psi do Bem. As produções de vídeos foram criadas com ferramentas de edição e tinham como objetivo os cuidados físicos e mentais, com: exercícios físicos, orientações referentes a vários tipos de patologias médicas, dança, música, meditação e alimentação saudável. Para o estudo foram incluídas as participações de mulheres grávidas que tiveram suas dúvidas esclarecidas. Professores e alunos apresentaram suas experiências no ensino remoto e discursaram sobre as emoções frente ao novo modelo de aulas, home office. A pesquisa também envolveu o segmento de profissionais ligados ao Recursos Humanos, ONGs e outras ações. Neste contexto, trocas afetivas foram intermediadas pelo apoio colaborativo da mediadora da rede. A Rede Psi do Bem se alimenta da troca de generosidade e empatia dos amigos do bem que se comprometem com o seu melhor.

As trocas foram observadas através da postagem em forma de emojis (Churches et al, 2014). Eles circulam no mundo transbordando os aplicativos de mensagens e cumprindo a função de transformar emoções em símbolos. Este fenômeno em plena pandemia possibilitou um estudo apoiado por ferramentas tecnológicas para entender como as imagens afetam os indivíduos. Para a psicologia o afeto é a capacidade do ser humano experienciar tendências, emoções, paixões e sentimentos (Conselho Federal de Psicologia, 2018). Através do afeto, revelamos nossos sentimentos e criamos laços de convivência.

\subsection{A Percepção dos Indivíduo}


O termo percepção é definido como "conhecimento de que algo existe, ou compreensão de uma situação ou assunto na atualidade com base em informação ou experiência" (Cambridge, 2020). A percepção leva o indivíduo a entender melhor as atividades que realiza e produzir resultados mais relevantes para o grupo (Vieira et al, 2009). A representação conhecida como framework $5 \mathrm{~W}+1 \mathrm{H}$, identifica seis questões básicas que devem ser respondidas quando se deseja ajudar um indivíduo a compreender algo sobre o qual ele não tem conhecimento prévio. A informação de percepção, portanto, é uma resposta a essas seis questões fundamentais, conforme descrito abaixo: (i) Quem informações sobre a presença e disponibilidade de indivíduos no grupo e identificação dos participantes envolvidos em um evento ou ação? (ii) $\mathrm{O}$ quê - informações sobre a ocorrência de um evento de interesse para o grupo. (iii) Onde - informações espaciais, de localização, o lugar onde o evento ocorreu. (iv) Quando - informações temporais sobre o evento, o momento em que o evento ocorreu. (v) Como - informações sobre como o evento ocorreu. (vi) Por quê - informações subjetivas sobre as intenções e motivações que levaram ao evento.

Existem duas formas de coletar as informações geradas pela percepção. Estas informações podem ser no modo síncrono ou assíncrono. No modo síncrono, os indivíduos interagem em eventos no mesmo espaço de trabalho compartilhado no momento atual, com notificações imediatas, disponibilidade para interação. Quanto à percepção no modo assíncrono, os participantes são informados sobre as interações que ocorreram antes do momento em que se conectam ao sistema. Para (Endley, 1995), existem três elementos principais de percepção, eles são: (i) Percepção da situação atual; (ii) Compreensão desta situação; e (iii) Projeção da condição futura. Para este estudo utilizaremos a percepção da situação atual e imediata, a partir das reações dos indivíduos que recebem os vídeos. Além da compreensão da situação atual, através da análise de postagem e interação dos grupos e o cenário de pandemia.

\subsection{Redes Colaborativas}

As redes colaborativas são definidas como um espaço em que os membros estão distribuídos geograficamente e, necessitam colaborar e cooperar para alcançar seus objetivos comuns a partir de ferramentas tecnológicas que possibilitam as conexões. (Islam et al., 2016).

As redes colaborativas possibilitam uma maior sinergia entre as áreas transdisciplinares, ampliando questões relacionadas aos problemas sociais que apoiem a construção de laços com seus pares e com o mundo real. Estas redes possibilitam a construção de um ambiente apoiado por colaboradores presenciais ou virtuais.

\section{Metodologia da pesquisa}

Este estudo foi conduzido ao longo de 9 meses - Março de 2020 até 20 de novembro de 2020 -, no qual foram produzidos 72 vídeos de diferentes temas, enviados diariamente. Estes vídeos tinham a duração de 5 a 10 minutos em média. Hoje existem mais de 500 seguidores entre Instagram e Facebook. A partir de agosto de 2020 o modelo de divulgação dos vídeos passou a ser 3 videos por semana. Para este estudo foram realizadas análises de resultados do Instagram e do Facebook. Na Tabela 1, apresentamos uma 
prévia da análise da coleta de dados realizada de acordo com as postagens nas redes sociais.

Tabela 1: Análise das Redes Sociais

\begin{tabular}{|l|l|l|}
\hline Análise das Redes Sociais & & \\
\hline Rede Social & Visualização & Curtida \\
\hline Instagram & 1724 & 1450 \\
\hline Facebook & 2900 & 2820 \\
\hline
\end{tabular}

Todos os vídeos produzidos foram construídos por especialistas voluntários que, na maioria das vezes, se candidataram para colaborar com o projeto fortalecendo a rede colaborativa. Na pesquisa contamos com 72 especialistas como: psicólogos, fisioterapeutas, médicos, endocrinologistas, ginecologistas, psiquiatras entre outros. Segue o link de um dos vídeos produzidos para Rede Psi do Bem: https://t.me/redepsidobem/144. Segue abaixo os passos utilizados para a análise do estudo.

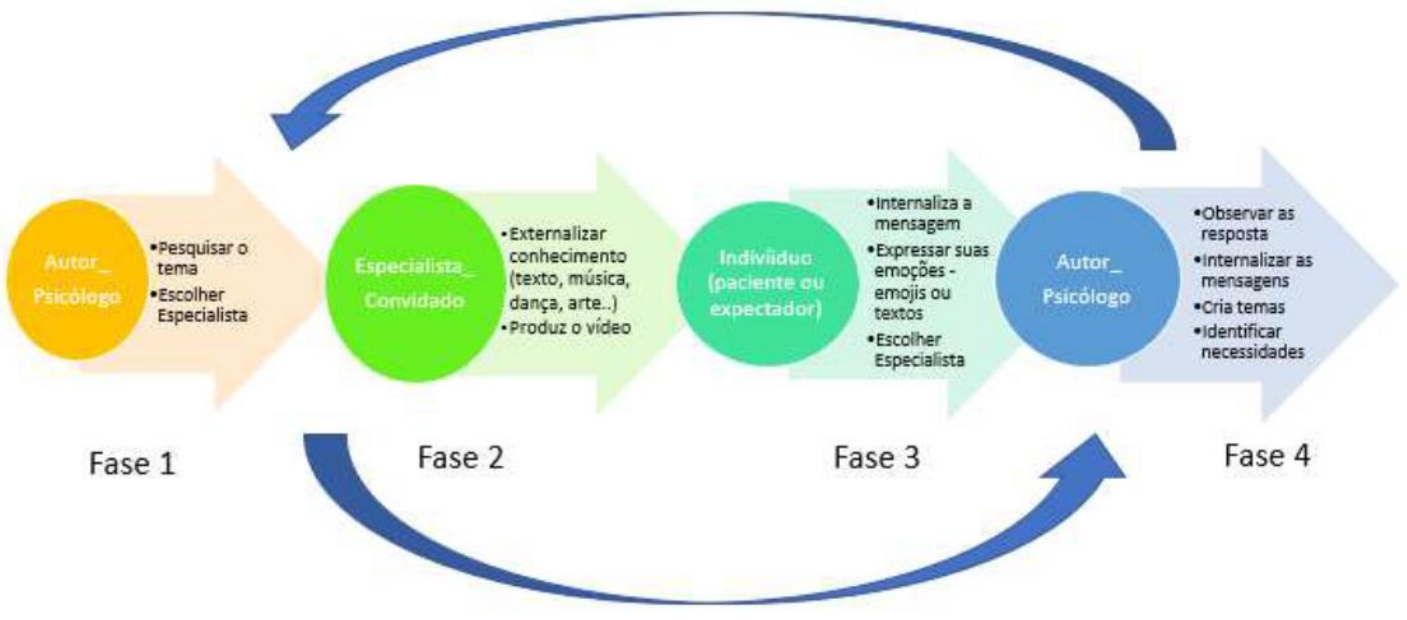

Figura 1: Fluxo da Rede Psi do Bem Fonte: Elaborada pelos autores

A Figura 1 apresenta o Fluxo de Construção da Rede Psi do Bem. Na Fase 1, o autor, psicólogo, pesquisa os temas de acordo com as informações de seus pacientes e amigos. Depois, os especialistas convidados contribuem com uma proposta de tema. Fase 2 , o especialista entende a proposta da Rede Psi do Bem e produz um vídeo - que pode ser pelo celular - sobre sua área de conhecimento e a relação do tema com a pandemia. A Fase 3 diz respeito ao indivíduo, paciente ou espectador, que assiste e internaliza o tema do vídeo. Em seguida, expressa ou não sua emoção (emojis ou texto) e pode sugerir um tema novo. Por último, o autor, psicólogo, observa as respostas, analisa o tipo de devolutiva, internaliza as informações capturadas das redes sociais e retorna a Fase 1, iniciando o novo fluxo da Rede Psi do Bem. 


\subsection{Discussões sobre os Resultados}

Os indivíduos recebem os vídeos pelas redes sociais Whatsapp, Instagram e Facebook. Observou-se que à medida que eram afetados pelo vídeo, os espectadores reagiram de forma diferente. A pesquisa de observação tinha como objetivo identificar os emojis $e$ mensagem de texto para tentar entender e inferir sobre as possíveis emoções. Os emojis são símbolos que representam emoções, que podem ser amor ( ), aplausos ( $\mathbb{0}$ ), raiva () etc. Durante a análise das postagens percebemos que grande parte indivíduos/seguidores retornavam com emojis e continuavam seguindo a Rede Psi do Bem. Além disso, repassavam os vídeos para outros indivíduos criando uma rede ainda maior e mais participativa. Em alguns casos, os seguidores usavam a mensagem privada para pedir ajuda para o psicólogo e algum outro especialista.

A análise dos emojis eram seguidas pelos autores, juntamente com as reações com relação aos temas. Para este estudo observamos, especificamente, a ferramenta Instagram $e$ Facebook. Neste momento, preferimos não trabalhar com o Whatsapp, pois a ferramenta não guarda os históricos da mensagem.

Durante as observações foram coletadas as percepções através de depoimentos dos participantes da Rede Psi do Bem. Os participantes traziam para o espaço da rede colaborativa novos indivíduos e relataram suas preocupações, angústias e impactos das informações divulgadas. Seguem alguns relatos dos participantes da rede, como: "Há muita falta de responsabilidade, e de conhecimento"; "Falta de amor ao próximo"; "Resiliência sempre!!"; "as barreiras físicas não impedirão de viver a sua vida"; "Vamos ter energia e força, para enfrentar estes tempos tão inseguros"; "Grata por estes momentos"; "adorei o esclarecimento tão oportuno, nesse momento"; "Obrigada, amei ouvi-la.", "Gratidão por nos ajudar a vencer este período mais levemente”, "Conteúdo rico, essencial para o momento....", ”... ficaria o dia inteiro ouvindo... ", "ouvir outros especialistas me conforta" $e$ "...saber que posso contar com a rede acalma meu coração e me fortalece...". Estes foram alguns dos trechos retirados dos depoimentos postados nas mídias sociais. Este estudo possibilitou apoiar muitas pessoas em situação de estresse. Também observamos que parte dos indivíduos/seguidores não apresentam reações positivas e negativas. Assim, acreditamos que existe grande necessidade de expandir a pesquisa para coletar mais informações.

\section{Conclusões}

Esta pesquisa desenvolvida ao longo de 9 meses possibilitou investigar o que o isolamento acrescentou ao atendimento individual dos psicólogos. A partir da quarentena os atendimento remotos aos pacientes foram impostos e muito necessários. Acompanhar os pacientes e manter a sanidade dos amigos e desconhecidos priorizou a criatividade com vídeos informativos. Para isso, buscamos responder a questão "Como o uso de mídias sociais, em uma rede colaborativa, podem apoiar a saúde mental dos indivíduos em situações de isolamento?".

O desafio de entender como usar as ferramentas tecnológicas trouxeram para o atendimento clínico um conjunto de possibilidades nunca antes pensado e permitido. Investigar os indivíduos pelos textos e seus emojis para entender as emoções em determinados contextos de interação foi um desafio necessário. Observamos as práticas sociais de linguagem acontecendo de forma complexa devido à inter-relação dos vários 
agentes e modos de produção de sentido e tecnologias que fazem a mediação. Ao mesmo tempo, provoca e deixa emergir sentidos acrescidos de muitos outros significados, especialmente, de emoção. Em algumas situações, as imagens representam fortes sentimentos de afeto e precisam ser interpretadas.

Nossa maior limitação foi romper a complexidade de entender a transdisciplinaridade de interagir com diferentes especialistas e indivíduos, tecnologias e conceitos novos advindos de diferentes áreas. Também tivemos que mobilizar um público mais idoso, muitas vezes sem experiência no uso de tecnologia para acompanhar os vídeos. No qual também encontramos um potencial de estudo.

Para trabalhos futuros pretendemos realizar uma pesquisa exploratória com um grupo maior de participantes e, assim, entender suas percepções com o uso das tecnologias utilizadas e as interações com esses meios. Também pretendemos realizar entrevistas com os especialistas e entender sua participação na rede e o impacto que gerou na sua vida pessoal e profissional.

\section{Referências bibliográficas}

CAMBRIDGE,

Org/dictionary.

Available

in https://dictionary.cambridge.org/dictionary/english/. Accessed date Oct, 24, 2020.WHO.

WORLD HEALTH ORGANIZATION. Discurso de abertura do Diretor- Geral da OMS no briefing para mídia sobre Covid-19, 11 de março de 2020.

CHURCHES, O. et al. Emoticons em mente um estudo potencial relacionado a eventos. Páginas 196-202, publicado online: 06 de jan 2014. https:// doi.org/10.1080/1740919.2013.873737

CONSELHO FEDERAL DE PSICOLOGIA. Resolução do Exercício Profissional N 4 Resolução N4,26 de Março de 2020. Essa resolução regulamenta os serviços psicológicos por meio de tecnologia da informação durante o período da Pandemia do Covid19 (Cetic)referente a 2018- (29 de jan 2020 ás 5:13pm) jornaldebrasilia.com.br>brasil.

DUAN, L.; ZHU, G. (2020) Psychological interventions for people affected by the Covid19 epidemic. The Lancet, 7, 300-302. https://doi.org/10.1016/S2215-0366(20)30073-0

ENDLEY, M.R "Toward a theory of situation awareness in dynamic systems". Human Factors, vol. 37, pp. 32_64, Sep. 1995.

IISLAM, M. J. et al. (2016, July). Implementation of an industrial visualization model for collaborative networks. In 2016 IEEE 14th International Conference on Industrial Informatics (INDIN) (pp. 720-725). IEEE.

KOSSEK, E. E. Managing work-life boundaries in the digital age. Organizational Dynamics, 45, 258-270. 2016.

LIMA, C. K. T. et al. ( 2020) . The emotional impact of coronavirus 2019-Ncov (new coronavirus Disease) Psychiatry Research,287, e 112915. https://doi.org/10.1016/j.psychres.2020.112915 
MALAN, D.H. (1983). Psicoterapia Individual e a Ciência da Psicodinâmica. Trad.Maria Clarissa Juchem - Porto alegre - Artes Médicas.

SÁ, S. R., BÚ, E., LIMA, N. A. . Manual de Diretriz para atenção psicológica nos Centro Regional de Estudo para o 'Desenvolvimento da sociedade da Informação hospitais em tempos de combate ao Covid19.Revista Saúde \& ciência online [internet] $2020 ; 8(2)$ : 1 24. Disponível em:https:/www.ccih.med.br/wp-content/uploads/2020/04/876-2447-2PB.pdf. Acesso em 11 de Jun de 2020.

SCHMIDT, B. et al. Saúde Mental e Intervenções Psicológicas diante da Pandemia do Novo Coronavírus ( Covid19). Campinas. vol.37. Campinas 2020.

STERNBERGH, A. (2014). Smile, You're speaking emoji: the rapid evolution of a wordless tongue. New York News \& Politics. Disponível em: $<$ http://nymag.com/daily/intelligencer/2014/11/emojis-rapid-evolution.html>. Acesso em 20 out. 2020.

VIEIRA, V., TEDESCO, P.; Salgado,A.C. Models and processes for the development of context-sensitive systems. Update Days in Informatics (JAI'09), cap8, p.381-431. Porto Alegre: UFRGS, Publishing Company SBC, 2009.

XIANG, Y. T. et al. Timely mental health care for the 2019 novel coronavirus mental outbreak needed. The Lancet Psychological 2020;7 (3) 228-229.

WINNICOTT, D. W. (1975): O Brincar \& a Realidade. Imago Editora LTDA. Coleção Psicologia Psicanálise - .direção Jayme Salomão

WINNICOTT, D. W. (1978) Textos Selecionados: da pediatria à psicanálise/ D>W Winicott; tradução [de] Jane Russo. Rio de Janeiro: F. Alves,1978 (Série Psicologia e Psicanálise). 